Kong. Res. J. 2(1) : 128-132, 2015

ISSN 2349-2694

Kongunadu Arts and Science College, Coimbatore.

\title{
PHYTOCHEMICAL ANALYSIS AND IN VITRO FREE RADICAL SCAVENGING ACTIVITIES OF MEDICAGO SATIVA SEEDS
}

\author{
Gomathi, R${ }^{1}$., S.Banu ${ }^{2}$ and K. Usha ${ }^{3}$ \\ ${ }^{1}$ Department of Biochemistry, ${ }^{2}$ Biotechnology and ${ }^{3}$ Bioinformatics \\ Avinashilingam Institute for Home Science and Higher Education for Women, Coimbatore.
}

\begin{abstract}
Phytochemical analysis and in vitro free radical scavenging activities were analyzed in the various extracts of Medicago sativa seeds. The phytochemical analysis showed the presence of alkaloids, carbohydrates, flavonoids, glycosides, saponins, phytosterols, tannins, terpenoids and phenols. Among the various extracts, phytochemicals were extracted best in ethanol. Free radical scavenging activities such as 2,2-diphenyl-1-picrylhydrazyl (DPPH), hydroxyl, superoxide, 2,2'-azino-bis (3-ethylbenzothiazoline-6sulphonic acid) (ABTS), ferrous ion chleating activity and non radicals such as hydrogen peroxide and nitric oxide were analyzed in the various extracts of Medicago sativa seeds and were compared with standard antioxidant ascorbic acid. All the extracts of Medicago sativa seeds scavenged the free radicals in a concentration dependent manner. The antioxidative activity of all the extracts was found to be more pronounced than that of the standard antioxidant ascorbic acid. Among the various extracts, the antioxidant activity was found to be more pronounced in ethanolic extract of Medicago sativa seeds.
\end{abstract}

Keywords: Medicago sativa, Alfalfa, phytochemicals, DPPH, ABTS, free radicals and antioxidant.

\section{INTRODUCTION}

Plant materials remain an important resource to combat serious diseases in the world. The traditional medicinal methods, especially the use of medicinal plants, still play a vital role to cover the basic health needs in the developing countries. The medicinal value of these plants lies in some chemical active substances that produce a definite physiological action on the human body. The most important bioactive constituents of plants are alkaloids, tannin, flavonoid and phenolic compounds (Edeoga et al., 2005).

Reactive oxygen species such as superoxide anions $\left(\mathrm{O}_{2}{ }^{\circ}\right)$, hydroxyl, nitric oxide radicals and hydrogen peroxide $\left(\mathrm{H}_{2} \mathrm{O}_{2}\right)$ play an important role in oxidative stress related to the pathogenesis of various important diseases (Halliwell and Gutteride, 1999; Finkel and Holbrook, 2000). Antioxidants act as a major defense against radical mediated toxicity by protecting the damages caused by free radicals. Antioxidant based drugs/formulations for the prevention and treatment of complex diseases, like atherosclerosis, stroke, diabetes, Alzheimer's disease and cancer, have appeared in the last three decades (Devasagayam et al., 2004).

Medicago sativa L., or Alfalfa is a member of Leguminosae or Fabaceae family, which has a long history of dietary and medicinal uses in traditional herbal medicine in China, America, India and many
Middle Eastern countries for the treatment of a variety of ailments and is also used as a fodder for animals (Al-Dosari, 2012). Medicago sativa L., sprouts are often consumed as vegetable salad and their leaves or seeds are also sold as bulk powdered herb, capsules and tablets for nutritional supplement in health food stores. The extracts from alfalfa sprouts, leaves and roots have been indicated to be helpful in lowering cholesterol levels in animal and human studies (Hong et al., 2009). In this regard, the present investigation was aimed to analyze the phytochemical constituents and free radical scavenging activity of $M$. sativa raw seeds and germinated seeds. The present study was aimed to analyze the phytochemical constituents and in vitro free radical scavenging activities of $M$. sativa seeds.

\section{MATERIALS AND METHODS}

\subsection{Plant material}

The M.sativa seeds were collected from Tamil Nadu Agricultural University, Coimbatore, Tamil Nadu, India and authenticated (BSI/SRC/5/23/2014-15/Tech/394) by the authority of the botanical survey of India (BSI), Tamil Nadu Agricultural University, Coimbatore, Tamil Nadu, India.

\subsection{Preparation of the plant sample}

After that, the seeds were washed with water to remove contamination and shade dried at room 
temperature. The dried samples were ground into coarse powder with the help of mechanical grinder and stored in airtight containers for further studies. 20 grams of powdered samples were weighed and wrapped separately with whatmann No.1 filter paper and extracted using various solvents such as petroleum ether, benzene, chloroform, ethyl acetate, ethanol, methanol and aqueous with the help of soxhlet extractor. Then, the various plant extracts were concentrated using rotary evaporator and then preserved for future analysis.

\subsection{Qualitative analysis of phytochemicals}

The phytochemical constituents of various extracts of Medicago sativa seeds were qualitatively analyzed using standard procedure (Iyengar, 1995; Siddiqui and Ali, 1997; Raaman, 2006).

\subsection{Determination of free radical scavenging activity}

Free radical scavenging activities such as DPPH, hydroxyl, hydrogen peroxide, nitric oxide, superoxide, ABTS and ferrous ion radical scavenging activities were analyzed using standard procedures (Carter, 1971; Misra and Fridovich, 1972; Green et al., 1984; Ruch et al., 1989; Elizabeth and Rao, 1990; Mensor et al., 2001; Shirwaiker et al., 2006.

\section{RESULTS AND DISCUSSION}

Table1 indicates the phytochemical analysis of Medicago sativa seeds. The alkaloids and flavonoids were found to be present in all the extracts including petroleum ether, benzene, ethanol, methanol and aqueous extracts. The anthraquinones were found to be present in all the extracts except petroleum ether and aqueous extract. Saponins were present in chloroform, ethanol and methanol extracts. Phenols were present in all the extracts except ethyl acetate. Steroids were present in petroleum ether, benzene, ethyl acetate, and aqueous extracts. Tannins were found in chloroform, ethyl acetate, ethanol and methanol extracts. Terpenoids were found in petroleum ether, chloroform, ethanol, methanol, and aqueous extracts. Glycosides were found to be present in ethanolic extract alone. Phenols were found to be present in petroleum ether, benzene and chloroform extracts. Saponins were found to be present in chloroform, ethanol and methanolic extracts. Steroids were found to be present in petroleum ether, benzene, ethyl acetate and aqueous extracts. Tannins were found to be present in chloroform, ethyl acetate, ethyl acetate, ethanol and methanolic extracts. Terpenoids were found to be present in all the extracts except benzene and ethyl acetate extracts.
Table 1: Qualitative analysis for the presence of phytochemicals.

\begin{tabular}{|l|c|c|c|c|c|c|c|}
\hline $\begin{array}{l}\text { Phytochemical } \\
\text { constituents }\end{array}$ & PE & B & C & EA & E & M & A \\
\hline Amino acids & - & - & - & + & + & + & - \\
\hline Anthraquinones & - & + & + & + & + & + & - \\
\hline Alkaloids & + & + & - & - & + & + & + \\
\hline Carbohydrates & + & + & - & + & + & - & - \\
\hline Flavonoids & + & - & + & - & + & + & + \\
\hline Glycosides & - & - & - & - & + & - & - \\
\hline Phenols & + & + & + & - & - & - & - \\
\hline Saponins & - & - & + & - & + & + & - \\
\hline Steroids & + & + & - & + & - & - & + \\
\hline Tannins & - & - & + & + & + & + & - \\
\hline Terpenoids & + & - & + & - & + & + & + \\
\hline
\end{tabular}

(+ Presence; - Absence, PE - Petroleum ether; B - Benzene; C Chloroform; EA - Ethyl acetate; E - Ethanol; M - Methanol, A Aqueous )

Among the various extracts, the ethanolic extract of Medicago sativa seeds showed maximum phytochemical constituents. So, the ethanolic extract of Medicago sativa seeds were taken for further study.

The phytochemical screening of $C$. lanatus seeds showed the presence of phytochemical constituents such as alkaloids, flavonoids, tannins, aminoacids, carbohydrates, cardioglycosides, terpenoids, oils and fats in the ethanolic extract of plant material when compared with other solvents (Varghese et al., 2013).

\subsection{Free radical scavenging activity}

The scavenging of stable DPPH radical model is a widely used method to evaluate antioxidant activities in a relatively short time compared with other methods. The effect of antioxidants on DPPH radical scavenging was thought to be due to their hydrogen donating ability. DPPH radical is a stable free radical and accepts an electron or hydrogen radical to become a stable diamagnetic molecule (Soares et al., 1997).

Figure 1 indicates the DPPH radical scavenging activity of various extracts of Medicago sativa seeds. Ethanolic extract of Medicago sativa seeds showed the highest DPPH scavenging activity which was followed by standard antioxidant ascorbic acid, methanol and chloroform extracts. This may due to the extraction of maximum phytochemicals in ethanolic extract. The least activity was noted in aqueous extract. 


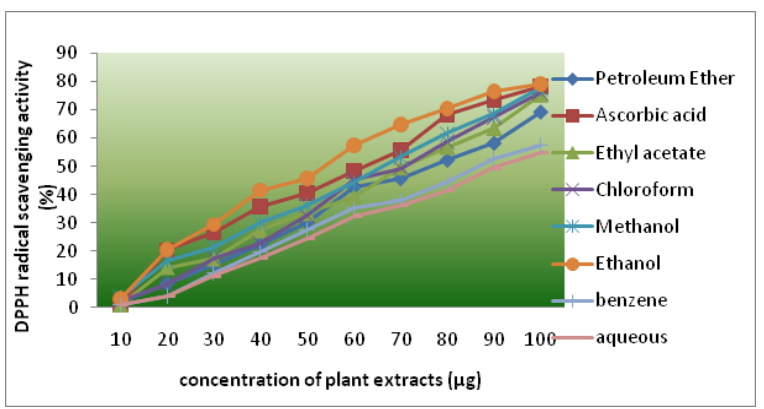

Fig. 1. Free radical scavenging activity of various extracta of $M$. sativa seeds by 2,2-diphenyl-1picryl hydrazyl radicals (DPPH).

\subsection{Hydroxyl radical scavenging activity}

The hydroxyl radical is an extremely reactive free radical formed in biological systems and has been implicated as a highly damaging species in free radical pathology capable of damaging the biomolecules of living cells (Zhang et al., 2009).

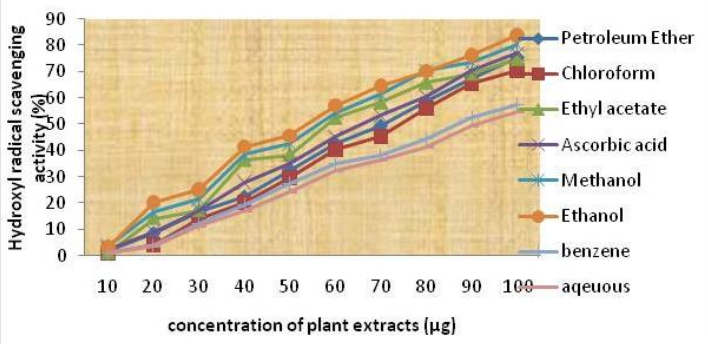

Fig. 2. Hydroxyl radical $(\mathrm{OH})$ scavenging effects of various extracts of $M$. sativa seeds.

Figure 2 illustrates the hydroxyl radical scavenging activity of various extracts of Medicago sativa seeds. Ethanolic extract of Medicago sativa seeds showed the highest radical scavenging activity which was followed by standard antioxidant ascorbic acid, methanol and ethyl acetate extracts. The least activity was noted in aqueous extract.

\subsection{Scavenging of hydrogen peroxides $\left(\mathrm{H}_{2} \mathrm{O}_{2}\right)$}



Fig. 3. Hydrogen peroxide (H202) scavenging effects of various extracta of $M$. sativa seeds.
$\mathrm{H}_{2} \mathrm{O}_{2}$ is highly important because of its ability of penetrate biological membranes. $\mathrm{H}_{2} \mathrm{O}_{2}$ itself is not very reactive, but it can sometimes be toxic to cell because it may give rise to hydroxyl radical in the cells. Thus, removing $\mathrm{H}_{2} \mathrm{O}_{2}$ is very important for the protection of living systems.

Figure 3 illustrates the hydrogen peroxide radical scavenging activity of various extracts of Medicago sativa seeds. Ethanolic extract of Medicago sativa seeds showed the highest radical scavenging activity which was followed by standard antioxidant ascorbic acid and methanol. The least activity was noted in aqueous extract.

\subsection{Nitric oxide scavenging activity}

Nitric oxide (NO) is a free radical produced in mammalian cells, involved in the regulation of various physiological processes. However, excess production of NO is associated with several diseases Nitric oxide is a very unstable species under aerobic condition. It reacts with $\mathrm{O}_{2}$ to produce stable product nitrate and nitrite through intermediates $\mathrm{NO}_{2}, \mathrm{~N}_{2} \mathrm{O}_{4}$ and $\mathrm{N}_{3} \mathrm{O}_{4}$ (Jayakumari et al., 2012).

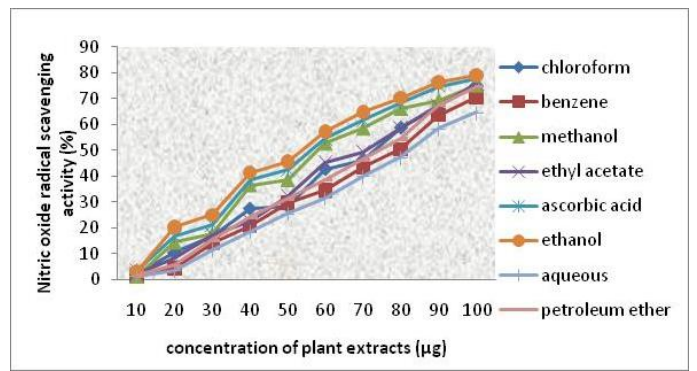

Fig. 4. Nitric oxide radical scavenging activity of various extracts of Medicago sativa seeds

Figure 4 illustrates the nitric oxide radical scavenging activity of various extracts of Medicago sativa seeds. Ethanolic extract of Medicago sativa seeds showed the highest radical scavenging activity which was followed by standard antioxidant ascorbic acid, methanol and ethyl acetate extracts. The least activity was noted in aqueous extract.

\subsection{Superoxide anion radical scavenging activity}

Superoxide anion radicals are produced endogenously by flavoenzymes like xanthine oxidase, which converts hypoxanthine to xanthine and subsequently to uric acid in ischemiareperfusion (Bora and Sharma, 2010). Superoxide is generated in vivo by several oxidative enzymes, including xanthine oxidase. In PMS-NADH-NBT system, superoxide anion derived from dissolved oxygen by PMS-NADH coupling reaction reduces NBT. The decrease of absorbance at $560 \mathrm{~nm}$ with 
antioxidants indicates the consumption of superoxide anion in the reaction mixture.

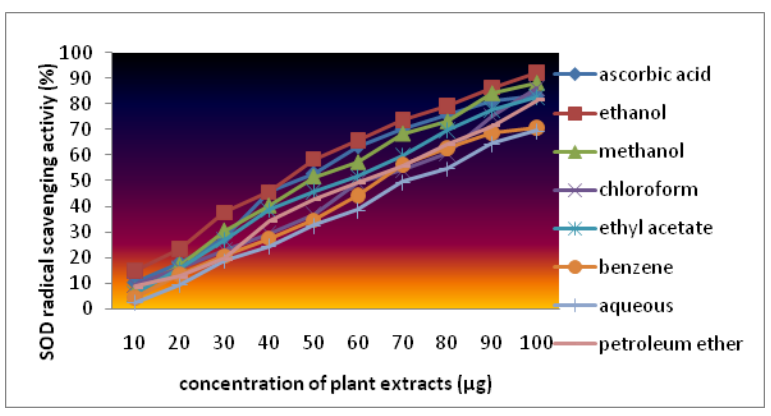

Fig. 5. superoxide anion radical scavenging activity of various extracts of Medicago sativa seeds

Figure 5 illustrates the inhibition of superoxide radical generation of various extracts of Medicago sativa seeds. Ethanolic extract of Medicago sativa seeds showed the highest radical scavenging activity which was followed by standard antioxidant ascorbic acid, methanol and ethyl acetate extracts. The least activity was noted in aqueous extract.

\subsection{ABTS radical scavenging activity}

ABTS is frequently used by the food industry and agricultural researcher to measure the antioxidant capacities of foods (Peiyuan et al., 2010). Proton radical scavenging is an important attribute of antioxidants. ABTS, a protonated radical, has characteristic absorbance maxima at $734 \mathrm{~nm}$ which decreases with the scavenging of the proton radicals. ABTS is a blue green chromogen and this assay is based on the ability of the antioxidants to scavenge long - life radical cation ABTS.

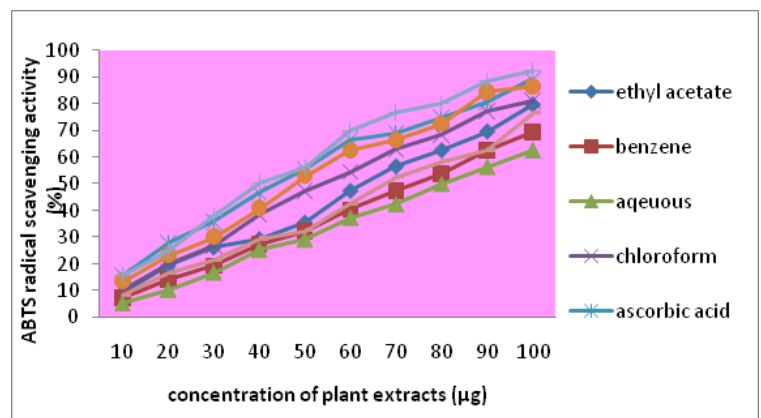

Fig. 6. ABTS radical scavenging activity of various extracts of Medicago sativa seeds.

Figure 6 illustrates the ABTS radical scavenging activity of various extracts of Medicago sativa seeds. Ethanolic extract of Medicago sativa seeds showed the highest radical scavenging activity which was followed by standard antioxidant ascorbic acid, methanol and ethyl acetate extracts.
The scavenging effect of all the extracts of Pouzolzia zeylanica increased with increasing concentration of Trolox has shown higher antioxidant activity (\% inhibition) in ABTS (2,2Azino-bis 3-ethyl benothiazoline-6-sulfonic acid diammonium salt) radical scavenging assay (Lobo et al., 2010).

\subsection{Ferrous metal ion chelating activity}

The chelating of ferrous ions by various extracts of Medicago sativa seeds were estimated by the method of Carter. Ferrozine can quantitatively form complexes with $\mathrm{Fe}^{2+}$. In the presence of chelating agents, the complex formation is disrupted with the result that the red color of the complex is decreased. Measurement of colour reduction, therefore, allows estimation of the chelating activity of the coexisting chelator. In this assay MMS and standard antioxidant compounds interfered with the formation of ferrous and ferrozine complex, suggesting that they have chelating activity and capture ferrous ion before ferrozine. Iron can stimulate lipid peroxidation by Fenton reaction, and also accelerates peroxidation by decomposing lipid hydroperoxides into peroxyl and alkoxyl radicals that can themselves abstract hydrogen and perpetuate the chain reaction of lipid peroxidation (Halliwell, 1991; Gulcin et al., 2003).

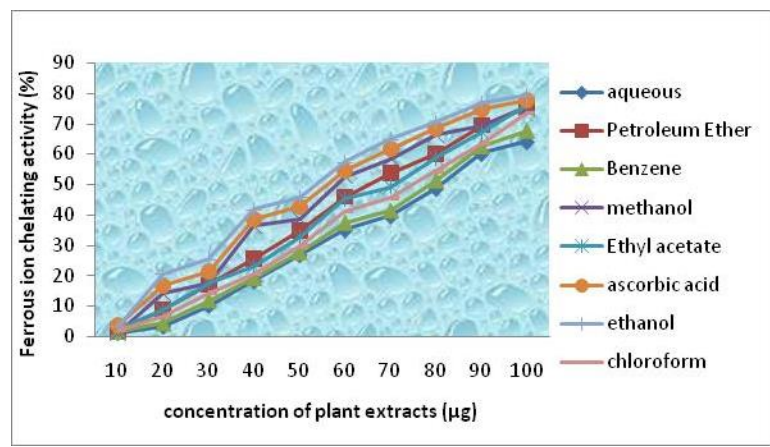

Fig. 7. Ferrous ion chleating activity of various extracts of Medicago sativa seeds.

Figure 7 illustrates the ferrous ion radical scavenging activity of various extracts of Medicago sativa seeds. Ethanolic extract of Medicago sativa seeds showed the highest radical scavenging activity which was followed by standard antioxidant ascorbic acid and methanol. The least activity was noted in aqueous extract.

\section{CONCLUSION}

From the results, it clearly indicates that $M$. sativa seeds possess powerful in vitro antioxidant activity. The encouraging results of various extracts $M$. sativa seeds with the various in vitro antioxidant tests proved the plant as a metal chelator, its 
hydrogen donating ability, and effectiveness as scavengers of free radicals, superoxide anions, hydroxyl and hydrogen peroxide. Further work will be carried out to isolate and elucidate the bioactive principle(s) responsible for the antioxidant activity.

\section{REFERENCES}

Al-Dosari, M.S. (2012). In vitro and in vivo antioxidant activity of Alfalfa (Medicago sativa L.)) on carbon tetrachloride intoxicated rats. The American J Chinese Med 40(4): 779-793.

Bora, K.S. and A. Sharma. (2010). In vitro antioxidant and free radical scavenging potential of Medicago sativa Linn. J Pharm Res 3(6): 12061210.

Carter, P. (1971). Spectrophotometric Determination of serum iron at the sub microgram level with a new regent - ferrozine. Anal. Biochem 40: 450458.

Devasagayam, T.P.A, J. Tilak, C. Boloor, K.K. Sane, K.S. Ghaskadbi and R.D. Lele. (2004). Review: Free radicals and antioxidants in human health: Current status and future prospects. J Assoc Phys India 52: 794-804.

Edeoga, H.O, D.E. Okwu and B.O. Mbaebie. (2005). Phytochemical constituents of some Nigerian medicinal plants. Afr J Biotech 4:685-688.

Elizabeth, K. and M.W.A. Rao. (1990). Oxygen radical scavenging activity of Curcumin. Int. J. Pharm. 58: $237-240$.

Finkel, T. and N.J. Holbrook. (2000). Oxidants, oxidative stress and the biology of aging. Nature 408: 239-247.

Green, M.J. and H.A.O. Hill. (1984). Chemistry of Dioxygen methods. Enzymology. 105.

Gulcin, I., M.E. Buyukokuroglu and O.I. Kufrevioglu. (2003). Metal chelating and hydrogen peroxide scavenging effects of melatonin. J. Pineal Res. 34: 278-281.

Halliwell, B. (1991). Reactive oxygen species in living systems: source, biochemistry and role in human disease. Am J Med. 91: 14-22.

Halliwell. B. and J.M.C. Gutteridge. (1999). Free radicals in biology and medicine. $3^{\text {rd }}$ ed., Oxford University Press, Oxford.

Hong, Y.H. W.W. Chao, M.L. Chen and B.F. Lin. (2009). Ethyl acetate extracts of alfalfa (Medicago sativa L.) sprouts inhibit lipopolysaccharide induced inflammation in vitro and in vivo. J Biomed Sci 16: 64 .
Iyengar, M.A. (1995). Study of crude drugs, $8^{\text {th }}$ edn Manipal power press.

Jayakumari, S., A. Arthanareswaran, A. Vijayalakshmi, M. Velraj and V. Ravichandran. (2012). Free radical scavenging activity of Pisonia grandis R.Br Leaves. Indian J Pharmaceu Edu Res 46(1): 34-37.

Lobo, V.C., A. Phatak and C. Naresh. (2010). Antioxidant and free radical scavenging activity of Hygrophila schulli (Buch.-Ham.) Almeida and Almeida Seeds. Advance in Bio Research 72-78.

Mensor, L.I., F.S. Menezes, G.G. Leitao, A.S. Reis, T. Dos- Santos, C.S. Coube and S.G. Leitao. (2001). Screening of Brazilian plant extracts for antioxidant activity by the use of DPPH free radical method. Phytotherapy Res 127-130.

Misra, H.P. and Fridovich. (1972). The role of superoxide anion in the antioxidation of epinephrine and a simple assay for superoxide dismutase. J. Biol. Chem., 247: 3170 - 3171.

Peiyuan, L., H. Lini, S. Wei, L. Rumei, D. Chaocheng, L. Liangquan, D. Yongkun, G. Nana, L. Chengsheng and H. Chunling. (2010). Free radicalscavenging capacity, antioxidant activity and phenolic content of Pouzolzia zeylanica. J Serbian Chem Soc 76(5): 709-717.

Raaman, N. (2006). Phytochemical Techiques. (New Publishing Agency), 19(24), 32-40.

Ruch, R.J., S.J. Cheng and J.E. Klaunig. (1989). Prevention of cytotoxicity and inhibition of intracellular communication by antioxidant catechins isolated from Chinese green tea. Carcinogen 10: 1003-1008.

Shirwaiker, A., H.N.A. Ram and F. Mohanpatra. (2006). Antioxidant and antiulcer activity of aqueous extract of a polyherbal formulation. Indian J. Exp. Bio. 44: 474-480.

Siddiqui, A.A. and M. Ali. (1997). Practical Pharmaceutical Chemistry, $1^{\text {st }}$ edn, CBS Publishers and Distributors, 126-131.

Soares, J.R. T.C.P. Dins, A.P. Cunha and L.M. Almeida. (1997). Antioxidant activity of some extracts of Thymus zygis. Free Radic Res. 26: 469-478.

Varghese, S., R. Narmadha, D. Gomathi, M. Kalaiselvi and K. Devaki. (2013). Phytochemical screening and HPTLC finger printing analysis of Citrullus lanatus (Thunb.) seed. J Acute Disease 122-126.

Zhang, W., B. Li, L. Han and H. Zhang. (2009). Antioxidant activities of extracts from Areca (Arecacatectu L.) flower, husk and seed. Afr J Biotechonol 8(16): 3887-3892. 